\title{
Construção de tecnologia leve como produto da disciplina segurança do paciente
}

\author{
Construction of light technology as a product of patient safety discipline
}

Construcción de tecnología de luz como producto de disciplina de seguridad para

pacientes

Patrícia da Costa Franco ${ }^{1 *}$, Daniela Sandy Silva de Souza1 ${ }^{1}$ Sara Nogueira Sampaio1, Bruna de Oliveira Moraes ${ }^{1}$, Hadelândia Milon de Oliveira1', Rizioléia Marina Pinheiro Pina1.

\section{RESUMO}

Objetivo: Descrever a experiência vivenciada por residentes do primeiro ano do Programa de Enfermagem Obstétrica de uma Universidade pública do Amazonas/Hospital Escola na construção de um Procedimento Operacional Padrão (POP) de identificação do paciente direcionado à área obstétrica. Relato de Experiência: Trata-se de um estudo descritivo, tipo relato de experiência. Para embasamento metodológico, utilizou-se o método de sistematização de experiências, proposto por Holliday OJ (2006), em que se descreve 1) O ponto de partida; 2) As perguntas iniciais; 3) Recuperação do processo vivido; 4) A reflexão a fundo; 5) Os pontos de chegada. Durante a construção do Procedimento Operacional Padrão (POP), foi possível vivenciar a experiência de trabalho em equipe, com reuniões para construção do protocolo de Identificação, interação entre os residentes com campo de discussões acerca da necessidade real da criação deste instrumento no cenário obstétrico. Considerações Finais: A experiência da elaboração de um POP no processo de formação da residência é vista como uma construção positiva, que leva á a reflexão dos residentes sobre processos necessários para a melhoria na qualidade do serviço de saúde. Destaca-se que o enfermeiro apresenta maior habilidade técnica, agregando cientificidade para desempenhar a aplicabilidade da Segurança do Paciente.

Palavras-chaves: Segurança do paciente, Tecnologia leve, Internato e residência, Enfermagem obstétrica.

\section{ABSTRACT}

Objective: Describe the experience of first year residents of the Obstetric Nursing Program of a Public University of Amazonas / Hospital Escola in the construction of a Standard Operating Procedure (SOP) for patient identification directed to the obstetric area. Experience Report: This is a descriptive study, type of experience report. For methodological basis, we used the method of systematization of experiences, proposed by Holliday OJ (2006), in which 1) The starting point is described; 2) The initial questions; 3) Recovery of the process experienced; 4) Deep reflection; 5) Arrival points. During the construction of the Standard Operating Procedure (SOP), it was possible to live the experience of teamwork, with meetings to build the Identification protocol, interaction between residents with a field of discussions about the real need to create this instrument in the obstetric scenario. Final Considerations: The experience of developing a SOP in the residency formation process is seen as a positive construction, which leads residents to reflect on processes necessary to improve the quality of the health service. It is noteworthy that the nurse has greater technical skill, adding scientificity to perform the applicability of patient safety.

Key words: Patient safety, Light technology, Pasantía y residencia, Obstetric nursing.

1Universidade Federal do Amazonas (UFAM), Manaus - AM. *E-mail: patriciacosta84@live.com 


\section{RESUMEN}

Objetivo: Describa la experiencia de los residentes de primer año del Programa de Enfermería Obstétrica de una Universidad Pública de Amazonas / Hospital Escola en la construcción de un Procedimiento Operativo Estándar (SOP) para la identificación del paciente dirigido al área obstétrica. Informe de Experiencia: Este es un estudio descriptivo, tipo de informe de experiencia. Como base metodológica, utilizamos el método de sistematización de experiencias, propuesto por Holliday OJ (2006), en el cual 1) Se describe el punto de partida; 2) Las preguntas iniciales; 3) Recuperación del proceso experimentado; 4) reflexión profunda; 5) Puntos de llegada. Durante la construcción del Procedimiento Operativo Estándar (SOP), fue posible experimentar la experiencia de trabajar en equipo, con reuniones para construir el protocolo de identificación, interacción entre residentes con un campo de discusión sobre la necesidad real de crear este instrumento en el escenario obstétrico. Consideraciones Finales: La experiencia de desarrollar un POE en el proceso de formación de residencia se considera una construcción positiva, que lleva a los residentes a reflexionar sobre los procesos necesarios para mejorar la calidad del servicio de salud. Es de destacar que la enfermera tiene una mayor habilidad técnica, agregando cientificidad para realizar la aplicabilidad de la seguridad del paciente.

Palabras clave: Seguridad del paciente, Tecnología ligera, Internado y residencia, Enfermería obstétrica.

\section{INTRODUÇÃO}

A portaria no 529 de $1^{\circ}$ de abril de 2013 institui o Programa Nacional de Segurança do Paciente que objetiva contribuir para a qualificação do cuidado em saúde em todos os estabelecimentos de saúde do território nacional estabelecendo apoio para elaboração de protocolos, guias e manuais de segurança do paciente (BRASIL, 2013). A abordagem inicial desse relato visa à reflexão acerca da segurança do paciente e da utilização de ferramentas para sua aplicabilidade no contexto da formação na residência multiprofissional. A residência multiprofissional em saúde é caracterizada como uma pós-graduação lato sensu, voltada para a educação em serviço e destinada às categorias profissionais que integram a área de saúde (BRASIL, 2005).

A residência multiprofissional determina carga horária de 60 horas semanais distribuídos em conteúdo teórico, teórico-prático e prática em campo. Os conteúdos teóricos, são abordados e trabalhados em disciplinas de eixo comum e disciplina do eixo específico. A disciplina Segurança do Paciente é transversal aos programas de residência da Universidade Federal do Amazonas (UFAM), que teve como proposta a elaboração de um Procedimento Operacional Padrão (POP) seguindo as Metas Internacionais de segurança do paciente e Protocolos de Segurança do Paciente instituído pelo Ministério da Saúde dos quais se destaca o de Identificação do Paciente (BRASIL, 2016).

O POP é descrito como um documento imprescindível para o exercício de qualquer tarefa com vistas a qualidade, eficiência e eficácia, obedecendo critérios técnicos e observando normas e legislação das áreas pertinentes. Os POP's servem de veículo para que as informações acerca dos diversos processos cheguem com segurança ao executor (EBSERH, 2014). Em se tratando de definições, o POP enquadra-se a uma tecnologia leve. Segundo Merhy EE (2005), por definição, a tecnologia leve se refere a relações de produção de vínculo, acolhimento e gestão dos processos de trabalho. Com isso, ressalta-se a importância da aplicação dessa ferramenta nas instituições de saúde, além da participação dos profissionais em sua elaboração, pois a prática profissional enriquece o conteúdo estabelecido em um POP, e a vivência hospitalar auxilia na adaptação da teoria à realidade vivenciada (ROSSI FR e LIMA MADS, 2005).

De acordo com Tase T, et al. (2018), a implementação do POP de identificação do paciente impacta diretamente na redução de eventos adversos na área obstétrica. O cenário obstétrico no Brasil é, em sua maioria, centrado nas maternidades e é um desafio reduzir os índices de morbimortalidade materno-neonatal. Em 2016 foram contabilizados cerca de 1.670 óbitos maternos, sendo indicadores sensíveis da qualidade na prestação dos serviços. Muitas dessas mortes são evitáveis do ponto de vista biomédico (DATASUS, 2016).

Tase $T$, et al. (2018), em seu estudo realizado em maternidade constatou que $86 \%$ dos nomes das mulheres apresentavam fonéticas similares, decorrentes do primeiro nome e do último sobrenome, 
ressaltando a importância da existência de protocolos de identificação do paciente bem estruturados, restringido dessa maneira a possibilidade de falhas. O risco advindo da fragilidade da etapa de identificação decorre de dados incoerentes, gerando possíveis erros com o uso do dispositivo em circunstâncias clínicas especiais, como transfusão de sangue e administração de medicamentos, recém-nascido entregue à mãe não correspondente (ERBSEH, 2017).

Nesse sentido, este relato justifica-se pela necessidade de reflexão sobre a importância da elaboração de um POP acerca da identificação segura do paciente no cenário obstétrico no processo de formação do residente em enfermagem obstétrica. Nessa perspectiva o objetivo desse manuscrito é descrever a experiência vivenciada por residentes do primeiro ano do Programa de Enfermagem Obstétrica de uma Instituição de Ensino Superior do Amazonas/Hospital Universitário na construção de um POP de identificação do Paciente direcionado à área obstétrica buscando destacar a importância da construção de tecnologias leves no contexto de ensino-aprendizagem e prática.

\section{RELATO DE EXPERIÊNCIA}

Trata-se de um estudo descritivo, tipo Relato de Experiência. Para embasamento metodológico, utilizouse o método de sistematização de experiências, proposto por Holliday OJ (2006), onde propõe cinco etapas para explanação da experiência vivida em uma ordem justificada: 1) O ponto de partida, é o início de todo o processo de sistematização no momento de vivência da experiência, procurou-se durante o processo realizar registros documentais da experiência como relatório descrevendo a atividade; 2) As perguntas iniciais, momento da definição e delimitação dos objetivos a fim de sistematizar a experiência, foi considerado a questão da identificação no processo de trabalho no serviço obstétrico e neonatal sugerido pela distribuição de temas na disciplina; 3) Recuperação do processo vivido, tratou-se da reconstrução da história e as informações foram ordenadas e descritas na mesma sequência dos acontecimentos durante a construção do POP; 4) A reflexão de fundo, nesta etapa procurou-se analisar e interpretar criteriosamente a experiência vivenciada por meio do embasamento teórico; 5) Os pontos de chegada, nesta etapa final foram formuladas as conclusões da experiência, evidenciando a relevância da vivência, bem como a comunicação do aprendizado por meio de explanação oral durante a disciplina Segurança do Paciente.

A experiência de construção do POP - Identificação do Paciente foi vivenciada durante a disciplina Segurança do Paciente, que compõe prioridade estratégica na matriz curricular do Programa de Residência Multiprofissional e Uniprofissional em Saúde de uma instituição de Ensino Superior do Amazonas/ Hospital Universitário, com carga horária de 30 horas. A disciplina teve por objetivo compartilhar e agregar conhecimentos sobre Segurança do Paciente, bem como levar os alunos a um pensamento reflexivo sobre práticas de saúde seguras. Dentre as propostas do processo de avaliação da disciplina, foi proposto aos residentes a construção de um POP visando sua aplicabilidade e viabilidade em unidades de saúde como protocolo de segurança do paciente.

Durante a disciplina foram elaborados e construídos quatro POP's, com temáticas diferentes, a saber, Lesão por Pressão; Prevenção de Quedas; Processos de Identificação de Pacientes; Checklist de Cirurgia Segura. Nesse contexto, as residentes do Programa de Enfermagem Obstétrica experienciaram elaborar estratégias para construção dos "Processos de Identificação de Pacientes" que tem por definição o processo pelo qual se assegura ao paciente sua identificação correta na realização de procedimentos e tratamentos, com objetivo de prevenir a ocorrência de erros e enganos que venham a provocar eventos adversos. Este foi desenvolvido para casas de parto e traz como membros da equipe executante os profissionais de saúde e técnicos administrativos.

Na explanação do POP em sala de aula foi utilizada uma abordagem dinâmica interativa com mediação pelo grupo expositor por meio de aula expositiva-dialogada e confecção de pulseiras de identificação para cada residente. A dinâmica realizada se consistiu em misturar as pulseiras de todos os residentes com finalidade de encontrar quatro participantes com mesma data de nascimento, para facilitar, cada programa de residência tinha uma cor de pulseira que os diferenciavam. Após a dinâmica, os residentes referiram que as cores facilitaram a identificação mais rápida, pois a cor identificava que pertenciam a um mesmo grupo, seguida dos dados contidos nas pulseiras para checagem das informações. 


\section{DISCUSSÃO}

A construção do POP de Identificação do Paciente possibilitou aos residentes a experiência do trabalho em equipe. Na primeira reunião, foi discutida a real necessidade da criação deste instrumento no cenário obstétrico, haja vista a observação de ausência de identificação ou existência de identificação incompleta das pacientes, durante, suas atividades práticas nas maternidades, Essa situação foi considerada fator colaborador para o surgimento e falhas na execução de procedimentos pela equipe, dentre eles, administração de medicamentos e outras ações de cuidado atribuídas aos profissionais de saúde.

O POP é visto como instrumento gerencial, que o enfermeiro pode utilizar, com finalidade de promover melhorias na qualidade da assistência, conferindo padronização das intervenções de enfermagem, devendo ser construído e discutido com a equipe, considerando a realidade local de cada setor/unidade, incentivando o aperfeiçoamento das atividades desenvolvidas (SALES CB, et al., 2018). Os produtos da utilização do POP comprovam sua efetividade como ferramenta moderna para uma assistência segura (HONÓRIO RPP e CAETANO JA, 2009).

Em relação à importância do POP na área de Identificação do Paciente no contexto da obstetrícia, estudos apontam que mulheres admitidas na unidade obstétrica possuem alto risco de erro de identificação devido similaridade de nomes e sobrenomes, sendo este problema reconhecido mundialmente por suas consequências desastrosas para pacientes, profissionais e estabelecimentos de saúde. Alguns autores destacam que muitas dessas consequências são potencialmente fatais e, aproximadamente, nove por cento (9\%) delas provocam danos temporários ou permanentes (TASE T, et al., 2018; ECRI INSTITUTE, 2016).

Além da dificuldade com a similaridade de nomes e sobrenomes, outras inadequações encontradas no serviço de saúde, como o não preenchimento dos cabeçalhos dos registros profissionais nos prontuários, os quais deveriam conter, minimamente, nome completo da paciente, data de nascimento e número do registro, potencializam as chances de danos às pacientes, constituindo-se uma realidade contrária aos pressupostos do código de ética para enfermeiros, que refere como função da enfermagem adotar medidas apropriadas para salvaguardar pessoas, famílias e comunidades" (NETO JUNIOR JG, 2016; CIE, 2017)

Considerando este panorama, a dinâmica da utilização das pulseiras de identificação em sala de aula, serviu de fio condutor para a discussão e reflexão entre os residentes acerca da identificação correta dos pacientes e ressaltou a recomendação do Protocolo de Identificação do Paciente do Ministério da Saúde acerca da utilização de pulseira com a presença de no mínimo dois identificadores que possibilitem o profissional confirmar os dados presentes nela com aqueles contidos no prontuário, sendo eles: nome completo do paciente e/ou data de nascimento e/ou número do registro no serviço de saúde e/ou nome completo da mãe (BRASIL, 2013).

A dinâmica possibilitou perceber por meio da observação assistemática o conhecimento limítrofe de residentes multiprofissionais acerca da segurança do paciente, pois os discursos apontavam uma abordagem superficial da temática discutida, o que difere das residentes de enfermagem que discutiam a temática com maior profundidade e conhecimento o que pode estar relacionado essa discussão ter iniciado ainda na formação.

Autores corroboram com os resultados obtidos de que enfermeiros dialogam sobre assistência segura ainda durante a formação, quando afirmam que os enfermeiros, são capacitados desde a graduação para realizar ações gerenciais e por atuarem na linha de frente do cuidado em saúde, devem apresentar conhecimentos e habilidades para identificar os erros e tomar as medidas adequadas para revertê-los, bem como preveni-los, visando uma assistência segura e qualificada (OLIVEIRA JLC, et al., 2017; SOUZA VS, et al., 2015).

Para Sales CB, et al. (2018), é necessário a educação permanente para profissionais de saúde, com investimento em processos educativos e ações que visem a ascensão na prestação de serviços de qualidade. A implementação de POP's possibilita que os profissionais executem ações de cuidados padronizadas baseadas nas melhores evidências científicas, além de facilitar as tomadas de decisões, conferindo maior segurança no cumprimento de suas tarefas diárias, a fim de garantir cuidado livre de efeitos indesejáveis no resultado final (ALMEIDA ML, et al., 2016; MIRANDA AL, et al., 2016; OLIVO VF, et al., 2013). 
A experiência da elaboração de um POP no processo de formação da residência em enfermagem obstétrica é vista como uma construção positiva, que envolve reflexão sobre processos necessários para a melhoria na qualidade e oferta dos serviços de saúde neste âmbito, haja vista a necessidade real da construção e efetivação dessa tecnologia no contexto da saúde materna e infantil, coadjuvando acertadamente na redução da morbimortalidade nesse grupo. O enfermeiro destaca-se como profissional que possui maior habilidade técnico-científico no desenvolvimento e aplicação de estratégias que contribuam de maneira assertiva para a otimização das atividades laborais, a exemplo apontamos as Metas Internacionais de Segurança do Paciente, Protocolos do Ministério da Saúde, bem como a construção de POP's, operando como peça fundamental em todo o processo.

Esta perspicácia dar-se por compreender a importância da realização do trabalho em equipe, bem como da execução efetiva das tarefas em conjunto, onde todos têm como objetivo comum propiciar quer seja a promoção, prevenção, cura e/ou reabilitação do paciente, promovendo o seu bem estar, com foco na realização de atribuições, tarefas e atividades em conjunto, elaborando consensos e resultados coletivos com vistas a promover melhoria no serviço de saúde. Considerando, portanto, o exposto e as experiências agregadas, além da finalidade didática, a disciplina possibilitou a produção palpável de uma tecnologia leve, com vistas à posterior implementação e execução do POP de Identificação do Paciente nas maternidades onde as residentes desenvolvem suas práticas, firmando parceria com os órgãos gestores. Dessa forma, impulsionando a possibilidade do desenvolvimento de novos protocolos que possam colaborar para o avanço nas práticas de saúde que envolva a referida população, atribuindo impacto assertivo para saúde pública do Amazonas.

\section{REFERÊNCIAS}

1. ALMEIDA ML, et al. Instrumentos gerenciais utilizados na tomada de decisão do enfermeiro no contexto hospitalar. Texto Contexto Enferm [Internet], 2011; 20 (spe):1317.

2. AMARAL EMS, et al. Percepções sobre o trabalho da equipe de enfermagem em serviço hospitalar de emergência de adultos. REME - Rev Min Enferm, 2017; 21e:1023.

3. BRASIL, Lei 11.129 de 30 de junho de 2005. Diário Oficial da União, 2005.

4. BRASIL. Ministério da Saúde. Anexo 02: protocolo de identificação do paciente [Internet]. Brasília (DF): Ministério da Saúde; 2013.

5. BRASIL, Ministério da Saúde. Segurança do Paciente - Protocolos Básicos de Segurança do Paciente [online], 2016.

6. CONSELHO INTERNACIONAL DE ENFERMEIRAS (os). Código de Ética do CIE para enfermeiras (os) [Internet]. Revisado 2012. [Tradução para o português realizada por Telma Ribeiro Garcia (PPGENF-UFPB ICN Accredited ICNP® Centre), com revisão de Maria Amélia de Campos Oliveira (EEUSP). [citado 2017 Jun 14].

7. DATASUS. Óbitos de mulheres em idade fértil e óbitos maternos no Brasil. 2016 [online]

8. EBSERH - Empresa Brasileira de Serviços Hospitalares - Ministério da Educação. Protocolo de Serviço do Hospital Universitário Dr Miguel Riet Corrêa Jr. Protocolo de identificação do paciente HU-FURG- Rio Grande, 2017.

9. ECRI INSTITUTE. Patient identification errors [Internet]. 2016 [cited 2017 Jun 14].

10. HOLLIDAY OJ. Para sistematizar experiências. 2. Ed. Brasilia: MMA, 2006; 128p.

11. HONÓRIO RPP, CAETANO JA. Elaboração de um protocolo de assistência de enfermagem ao paciente hematológico: relato de experiência. Rev Eletr Enf [Internet], 2009; 11(1):188-93.

12. MERHY EE. Saúde: a cartografia do trabalho vivo. $2^{a}$ ed. São Paulo: Hucitec; 2005.

13. MIRANDA AL, et al. Resultados da implementação de um protocolo sobre a incidência de Infecção do Trato Urinário em Unidade de Terapia Intensiva. Rev Latino-Am Enferm [Internet], 2016.

14. NETO JUNIOR JG. Ciclo de melhoria para uma correta identificação do paciente em hospitais oncológicos [tese]. Natal: Universidade Federal do Rio Grande do Norte; 2016.

15. OLIVEIRA JLC, et al. Segurança do paciente: conhecimento entre residentes multiprofissionais. Rev. Einstein, 2017; 15(1): 50-7.

16. OLIVO VF, et al. Gerenciamento do processo de trabalho em enfermagem: um estudo diagnóstico para subsidiar a instituição de padrões de qualidade no serviço hospitalar. Bibl Lascasas [Internet]. 2013; 9(1).

17. ROSSI FR, LIMA MADS. Acolhimento: tecnologia leve nos processos gerenciais do enfermeiro. Rev. bras. enferm. 2005; 58(3).

18. SALES CB, et al. Protocolos Operacionais Padrão na prática profissional da enfermagem: utilização, fragilidades e potencialidades. Rev Bras Enferm [Internet]. 2018;71(1):138-46.

19. SOUZA VS, et al. Erros and adverse events: the interface with health professionals' safety culture. Cogitare Enferm. 2015;20(3):474-81.

20. TASE T, et al. Avaliação do risco de erro na identificação de mulheres numa maternidade pública. Rev. Bras. Enferm. 2018; 71(1): 120-5. 